\title{
Inverse relationship between serum osteocalcin levels and nonalcoholic fatty liver disease in postmenopausal Chinese women with normal blood glucose levels
}

\author{
Yu-qi LUO\#, Xiao-jing MA*, Ya-ping HAO, Xiao-ping PAN, Yi-ting XU, Qin XIONG, Yu-qian BAO*, Wei-ping JIA
}

Department of Endocrinology and Metabolism, Shanghai Jiao Tong University Affiliated Sixth People's Hospital; Shanghai Clinical Center for Diabetes; Shanghai Key Clinical Center for Metabolic Disease; Shanghai Diabetes Institute; Shanghai Key Laboratory of Diabetes Mellitus, Shanghai 200233, China

\begin{abstract}
Aim: Osteocalcin is involved in the progression of nonalcoholic fatty liver disease (NAFLD) in animal models and humans. In this study we investigated the relationship between serum osteocalcin levels and NAFLD in postmenopausal Chinese women.

Methods: A total of 733 postmenopausal women (age range: 41-78 years) with normal blood glucose levels were enrolled in this cross-sectional study. Women taking lipid-lowering or anti-hypertensive drugs were excluded. Serum osteocalcin levels were assessed using an electrochemiluminescence immunoassay. The degree of NAFLD progression for each subject was assessed through ultrasonography. The fatty liver index (FLI) of each subject was calculated to quantify the degree of liver steatosis.

Results: The median level of serum osteocalcin for all subjects enrolled was $21.99 \mathrm{ng} / \mathrm{mL}$ (interquartile range: $17.84-26.55 \mathrm{ng} / \mathrm{mL}$ ). Subjects with NAFLD had significantly lower serum osteocalcin levels $(18.39 \mathrm{ng} / \mathrm{mL}$; range: $16.03-23.64 \mathrm{ng} / \mathrm{mL}) \mathrm{compared} \mathrm{with} \mathrm{those}$ without NAFLD $(22.31 \mathrm{ng} / \mathrm{mL}$; range: $18.55-27.06 \mathrm{ng} / \mathrm{mL} ; P<0.01)$. Serum osteocalcin levels decreased with incremental changes in the FLI value divided by the quartile ( $P$-value for trend<0.01). The serum osteocalcin levels showed a negative correlation with the FLI values, even after adjusting for confounding factors (standardized $\beta=-0.124 ; P<0.01$ ). Binary logistic regression analysis identified an individual's serum osteocalcin level as an independent risk factor for NAFLD (odds ratio: 0.951; 95\% confidence interval: 0.9110.992; $P=0.02$ ).

Conclusion: Serum osteocalcin levels are inversely correlated with NAFLD in postmenopausal Chinese women with normal blood glucose levels.
\end{abstract}

Keywords: osteocalcin; nonalcoholic fatty liver disease; postmenopause; Chinese women

Acta Pharmacologica Sinica (2015) 36: 1497-1502; doi: 10.1038/aps.2015.81; published online 16 Nov 2015

\section{Introduction}

The liver plays a crucial role in regulating the levels of various molecules and energy production. Nonalcoholic fatty liver disease (NAFLD) is a disorder characterized by excessive accumulations of fat in liver hepatocytes and is associated with a variety of metabolic disorders. Furthermore, due to its high prevalence, NAFLD has triggered much public concern. Osteocalcin is a protein that is specifically synthesized and secreted by osteoblasts, and osteoblast function can be evaluated by measuring serum osteocalcin concentrations. More-

\footnotetext{
\# These authors contributed equally to this work.

* To whom correspondence should be addressed. E-mail byq522@163.com

Received 2015-05-14 Accepted 2015-08-11
}

over, osteocalcin has recently been shown to play an important role in regulating energy metabolism ${ }^{[1,2]}$.

In animal studies, osteocalcin treatment protected against high-fat diet-induced insulin resistance and substantially reduced the pathological signs of nonalcoholic steatohepatitis (eg, ballooning degeneration, steatosis, and fibrosis). Furthermore, osteocalcin produced an overall reduction in NAFLD activity scores ${ }^{[3]}$. Serum osteocalcin levels in humans are closely associated with the presence or absence of NAFLD. Yilmaz et al have reported that compared with control subjects, patients with biopsy-proven NAFLD had lower serum concentrations of osteocalcin and that serum osteocalcin concentrations were significantly associated with both serum transaminase activity and the extent of hepatocyte ballooning $^{[4]}$. Additionally, a community-based study of Chinese men 
found a negative correlation between serum osteocalcin levels and the presence of NAFLD, as diagnosed by ultrasonography ${ }^{[5]}$.

Serum osteocalcin concentrations are influenced by gender, as well as menopausal status among women ${ }^{[6]}$; however, few studies have examined the relationship between osteocalcin and NAFLD in women. A recent study has shown that serum osteocalcin levels in Korean women were independently and negatively correlated with NAFLD ${ }^{[7]}$; however, several confounding factors present in the study participants (e.g., glucose metabolism disorders and relevant medications) were not fully considered. To the best of our knowledge, no previous study has focused on the relationship between serum osteocalcin levels and NAFLD in Chinese women. Menopause and its accompanying estrogen loss greatly increase the risk for metabolic disorders in women. Therefore, to explicitly examine the relationship between serum osteocalcin levels and NAFLD in Chinese postmenopausal women, women with hyperglycemia or women who were undergoing medical treatments or taking medications were excluded from this study.

\section{Materials and methods}

\section{Study subjects}

All participants in this cross-sectional study were selected from individuals who were previously enrolled in the Shanghai Obesity Study (SHOS), which was conducted from December 2009 to December 2011 ${ }^{[8]}$. Each participant completed a questionnaire designed to gather demographic and lifestyle information as well as information regarding the subject's past medical history and current use of medications. All study participants had normal blood glucose levels, as defined by the following World Health Organization 1999 criteria: a fasting plasma glucose (FPG) level $<6.1 \mathrm{mmol} / \mathrm{L}$ and a 2 -h plasma glucose (2hPG) level $<7.8 \mathrm{mmol} / \mathrm{L}^{[9]}$. Subjects taking any medication that would likely affect the progression of NAFLD and/ or serum osteocalcin concentrations (e.g., anti-hypertensives, glucocorticoids or lipid lowering drugs) were excluded from the study. Additionally, individuals with a history of malignancy, stroke, cardiovascular disease, renal dysfunction, and hypo- or hyperthyroidism, as well as individuals with an active infectious condition or who were current tobacco or alcohol users were also excluded from the study. The history of menopause for each woman was determined through the questionnaire. Menopause is defined as at least 12 consecutive months of amenorrhea, without other medical causes for amenorrhea. Finally, a total of 733 postmenopausal women who provided complete eligibility data were enrolled in the study, and their results were included in our final analysis. The study methods and potential risks were fully explained to all subjects, and each subject provided written informed consent prior to completing the survey. The study protocol was reviewed and approved by the Ethics Committee of Shanghai Jiao Tong University Affiliated Sixth People's Hospital, and all procedures were performed in accordance with the Declaration of Helsinki.

\section{Anthropometric and laboratory measurements}

Each subject received a physical examination that included height, weight, waist circumference, and blood pressure measurements. Body mass index (BMI) was defined as the subject's weight in kilograms divided by the square of their height in meters. A fasting blood sample was collected from each subject after $10 \mathrm{~h}$ of overnight fasting, and a 2-h post-load blood sample was collected after a 75-g oral glucose tolerance test for use in various laboratory assays. Anthropometric and laboratory measurements were obtained using standard techniques, as previously described ${ }^{[10,11]}$. The biochemical blood indices analyzed in this study included measurements of FPG, 2hPG, glycated hemoglobin ( $\mathrm{HbA1c})$, serum fasting insulin (FINS), serum total cholesterol (TC), serum triglyceride (TG), high-density lipoprotein cholesterol (HDL-C), low-density lipoprotein cholesterol (LDL-C), serum alanine aminotransferase (ALT), aspartate aminotransferase (AST), gammaglutamyl transpeptidase (GGT), and C reactive protein (CRP). The homeostasis model assessment as an index of insulin resistance (HOMA-IR) was defined as FPG $(\mathrm{mmol} / \mathrm{L}) \times$ FINS $(\mathrm{mU} / \mathrm{L}) / 22.5$. Overnight fasting venous blood specimens were obtained to test for serum osteocalcin concentrations. Total osteocalcin levels were measured using an electrochemiluminescence immunoassay (Roche Diagnostics, $\mathrm{GmbH}$, Mannheim, Baden-Württemberg, Germany) on Roche Elecsys 2010 (Elecsys module) immunoassay analyzer, with calculated intra-assay and inter-assay coefficients of variation of $1.2 \%$ to $4.0 \%$ and $1.7 \%$ to $6.5 \%$, respectively ${ }^{[8]}$.

\section{Ultrasonography}

All participants received an abdominal ultrasonographic examination using a Voluson 730 Expert B-mode ultrasonogram device (GE Healthcare, 5.0-MHz transducer, Waukesha, WI, USA). All examinations were performed by the same experienced ultrasonographer, who was blinded to each subject's medical/demographic information and test results ${ }^{[10]}$.

\section{Diagnosis of NAFLD}

NAFLD was defined based on the criteria used in the 2010 revised guidelines for the management of NAFLD, published by the Chinese Hepatopathy Association ${ }^{[12]}$. Because we were unable to perform liver biopsies in this study, NAFLD was diagnosed based on images obtained during an ultrasound examination of the liver. Diffuse fatty liver was diagnosed when no other diagnosis could explain a subject's clinical signs and symptoms. Subjects with the following liver diseases were excluded from the study because their clinical findings might have interfered with the NAFLD diagnosis: viral hepatitis, alcohol- or drug-induced liver disease, total alcohol intake levels of $70 \mathrm{~g}$ per week, total parenteral nutrition-induced steatosis, autoimmune liver disease, and hepatolenticular degeneration. The fatty liver index (FLI) for each subject was calculated using the following equation to quantitatively assess the degree of liver steatosis:

$\mathrm{FLI}=\left(\mathrm{e}^{0.953 \times \log (\mathrm{TG})+0.139 \times \mathrm{BMI}+0.718 \times \log \mathrm{e}(\mathrm{GGT})+0.053 \times \text { waist circumference- } 15.745}\right) /(1+$ $\left.\mathrm{e}^{0.953 \times \log (\mathrm{TG})+0.139 \times \text { BMI }+0.718 \times \log (\mathrm{GGT})+0.053 \times \text { waist circumference- } 15.745}\right) \times 100^{[13]}$. 


\section{Statistical analyses}

All statistical analyses were performed using SPSS (Statistical Package for the Social Sciences) for Windows, Version 16.0 (SPSS Inc, Chicago, IL, USA). Normally distributed data are expressed as the mean value \pm standard deviation (SD), and data with a skewed distribution are presented as the median value with an interquartile range. An independent samples $t$-test and Mann-Whitney $U$-tests were used to compare differences in normally distributed data and skewed data, respectively, when searching for differences in clinical parameters in the NAFLD and non-NAFLD groups. One-way ANOVA was used for trend analyses. A linear regression analysis was performed to examine the independent relationships between the serum osteocalcin levels and FLI values. Binary logistic regression analysis was performed to identify the independent risk factors for NAFLD, and $P$-values $<0.05$ were considered to be statistically significant.

\section{Results}

Serum osteocalcin levels were significantly lower in NAFLD subjects

The anthropometric and metabolic characteristics of all study participants are summarized in Table 1. A total of 733 postmenopausal women (mean age, $56.1 \pm 4.5$ years; range, $41-78$ years), were enrolled in the study; among these women, 130 $(17.7 \%)$ were diagnosed with NAFLD. For all subjects, the median duration of menopause was 6 years (interquartile range, 3-10 years). The median serum osteocalcin level for all subjects was $21.99 \mathrm{ng} / \mathrm{mL}$ (interquartile range, 17.84-26.55 $\mathrm{ng} / \mathrm{mL}$ ). The correlation analysis showed that the serum osteocalcin levels were positively correlated with the duration of menopause $(r=0.089, P=0.016)$.

The subjects with NAFLD had significantly lower serum osteocalcin levels than the subjects without NAFLD (18.39 $\mathrm{ng} / \mathrm{mL}$ [range, 16.03-23.64 ng/mL] vs $22.31 \mathrm{ng} / \mathrm{mL}$ [range, 18.55-27.06 ng/mL], P<0.001). Additionally, compared to the non-NAFLD subjects, the subjects with NAFLD had significantly higher values for BMI, waist circumference, systolic blood pressure (SBP), blood glucose (FPG, 2hPG, and HbA1c), FINS, HOMA-IR, blood lipids (TC, TG, and LDL-C), liver enzymes (ALT, AST, and GGT), CRP, and FLI, but they had lower HDL-C levels (all $P$-values<0.05).

\section{Correlation between serum osteocalcin levels and FLI values}

The FLI values were divided into four groups, according to the quartile values (Q1, FLI<6.34, $n=183$; Q2, 6.34 $\leq \mathrm{FLI}<12.86$, $n=183 ; \mathrm{Q} 3,12.86 \leq \mathrm{FLI}<26.94, n=184 ; \mathrm{Q} 4, \mathrm{FLI} \geq 26.94, n=183$ ). Our trend analysis revealed a decreasing trend in serum osteocalcin levels, which was accompanied by increases in the FLI quartile (Q1, $23.20 \mathrm{ng} / \mathrm{mL}$ [range, 18.84-27.38 ng/mL]; Q2, $23.01 \mathrm{ng} / \mathrm{mL}$ [range, 19.00-27.41 ng/mL]; Q3, $22.19 \mathrm{ng} / \mathrm{mL}$ [range, 18.24-26.06 ng/mL]; Q4, $19.26 \mathrm{ng} / \mathrm{mL}$ [range, 16.19_

Table 1. General characteristics of the study subjects.

\begin{tabular}{|c|c|c|c|c|}
\hline Variables & Total & non-NAFLD & NAFLD & $P$ \\
\hline$n$ & 733 & 603 & 130 & - \\
\hline Age (year) & $56.1 \pm 4.5$ & $56.2 \pm 4.4$ & $56.0 \pm 5.0$ & 0.703 \\
\hline $\mathrm{BMI}\left(\mathrm{kg} / \mathrm{m}^{2}\right)$ & $22.7 \pm 2.9$ & $22.1 \pm 2.5$ & $25.5 \pm 2.8$ & $<0.001$ \\
\hline Waist circumference $(\mathrm{cm})$ & $78.1 \pm 8.0$ & $76.4 \pm 7.3$ & $85.7 \pm 6.9$ & $<0.001$ \\
\hline $\mathrm{SBP}(\mathrm{mmHg})$ & $120.0(110.0-129.3)$ & $120.0(110.0-128.0)$ & $120.3(113.3-130.0)$ & 0.015 \\
\hline DBP (mmHg) & $75.3(69.7-80.0)$ & $74.7(69.3-80.0)$ & $77.7(71.3-80.7)$ & 0.062 \\
\hline FPG (mmol/L) & $5.2 \pm 0.4$ & $5.1 \pm 0.4$ & $5.3 \pm 0.4$ & $<0.001$ \\
\hline $2 \mathrm{hPG}(\mathrm{mmol} / \mathrm{L})$ & $6.1 \pm 1.0$ & $6.0 \pm 1.0$ & $6.4 \pm 1.0$ & $<0.001$ \\
\hline $\mathrm{HbA} 1 \mathrm{c}(\%)$ & $5.6(5.4-5.8)$ & $5.6(5.4-5.8)$ & $5.7(5.5-5.9)$ & 0.038 \\
\hline FINS (mU/L) & $6.7(4.9-9.1)$ & $6.3(4.5-8.1)$ & $9.9(7.3-13.1)$ & $<0.001$ \\
\hline HOMA-IR & $1.6(1.1-2.1)$ & $1.4(1.0-1.9)$ & $2.3(1.7-2.9)$ & $<0.001$ \\
\hline $\mathrm{TC}(\mathrm{mmol} / \mathrm{L})$ & $5.54 \pm 0.95$ & $5.50 \pm 0.93$ & $5.74 \pm 1.02$ & 0.011 \\
\hline $\mathrm{TG}(\mathrm{mmol} / \mathrm{L})$ & $1.18(0.85-1.59)$ & $1.09(0.80-1.45)$ & $1.58(1.22-2.28)$ & $<0.001$ \\
\hline $\mathrm{HDL}-\mathrm{C}(\mathrm{mmol} / \mathrm{L})$ & $1.61 \pm 0.35$ & $1.66 \pm 0.35$ & $1.42 \pm 0.26$ & $<0.001$ \\
\hline LDL-C (mmol/L) & $3.44 \pm 0.82$ & $3.39 \pm 0.81$ & $3.63 \pm 0.86$ & 0.003 \\
\hline $\operatorname{ALT}(\mathrm{U} / \mathrm{L})$ & $16.0(12.0-21.0)$ & $15.0(12.0-20.0)$ & $21.5(16.0-28.0)$ & $<0.001$ \\
\hline AST (U/L) & $21.0(18.0-24.0)$ & $20.0(18.0-24.0)$ & $22.0(19.0-27.0)$ & 0.001 \\
\hline GGT (U/L) & $20.0(15.0-27.0)$ & $19.0(15.0-25.0)$ & $25.0(19.0-35.3)$ & $<0.001$ \\
\hline $\mathrm{CRP}(\mathrm{mg} / \mathrm{L})$ & $0.6(0.3-1.2)$ & $0.5(0.3-1.0)$ & $1.2(0.7-2.2)$ & $<0.001$ \\
\hline Osteocalcin (ng/mL) & $21.99(17.84-26.55)$ & $22.31(18.55-27.06)$ & $18.39(16.03-23.64)$ & $<0.001$ \\
\hline FLI & $12.86(6.34-26.94)$ & $10.69(5.42-19.49)$ & $40.32(24.42-52.15)$ & $<0.001$ \\
\hline
\end{tabular}

Data are means \pm standard deviation or median (interquartile range).

Abbreviation: 2hPG, 2-h plasma glucose level; ALT, alanine aminotransferase; AST, aspartate aminotransferase; BMI, body mass index; CRP, C reactive protein; DBP, diastolic blood pressure; FINS, fasting insulin; FLI, fatty liver index; FPG, fasting plasma glucose; GGT, gamma-glutamyl transpeptidase; HbA1c, glycated hemoglobin; HDL-C, high-density lipoprotein cholesterol; HOMA-IR, homeostasis model assessment-insulin resistance; LDL-C, lowdensity lipoprotein cholesterol; SBP, systolic blood pressure; TC, total cholesterol; TG, triglyceride. 
$23.74 \mathrm{ng} / \mathrm{mL}]$; $P$-value for the trend $<0.01$ ) (Figure 1 ).

We performed a linear regression analysis using FLI as the dependent variable to further examine the relationship between the FLI values and serum osteocalcin levels. When using only the serum osteocalcin level as the independent variable, our analysis identified a significant negative correlation between the serum osteocalcin levels and FLI values $(\beta=-0.560, P<0.001)$. Furthermore, this negative correlation remained significant after adjusting for age, SBP, diastolic blood pressure (DBP), blood glucose (FPG, 2hPG, and HbA1c), HDL-C, LDL-C, HOMA-IR, and CRP levels (standardized $\beta=-0.124 ; P<0.01$ ) (Table 2).

\section{Relationship between serum osteocalcin levels and NAFLD}

Next, we performed a binary logistic regression analysis, in which the presence of NAFLD was designated as the dependent variable in four different models (unadjusted; adjusted for age, BMI, and waist circumference; adjusted for liver enzymes; and adjusted full variables) to examine the relationship between the serum osteocalcin levels and NAFLD. Using the full model, the independent variables included age, BMI, waist circumference, osteocalcin, FPG, 2hPG, HbA1c, SBP, DBP, TG, HDL-C, LDL-C, ALT, AST, GGT, HOMA-IR, and CRP. However, the results showed that regardless of which model was used, a subject's serum osteocalcin level remained the independent variable that was most highly correlated with the presence of NAFLD (Table 3).

\section{Discussion}

NAFLD is the most common chronic liver disorder worldwide, and its occurrence is associated with several metabolic risk factors, including diabetes mellitus, dyslipidemia, and hypertension. Therefore, NAFLD is associated with increased rates of mortality from both cardiovascular-related and liverrelated causes ${ }^{[14,15]}$. Although proper and timely disease

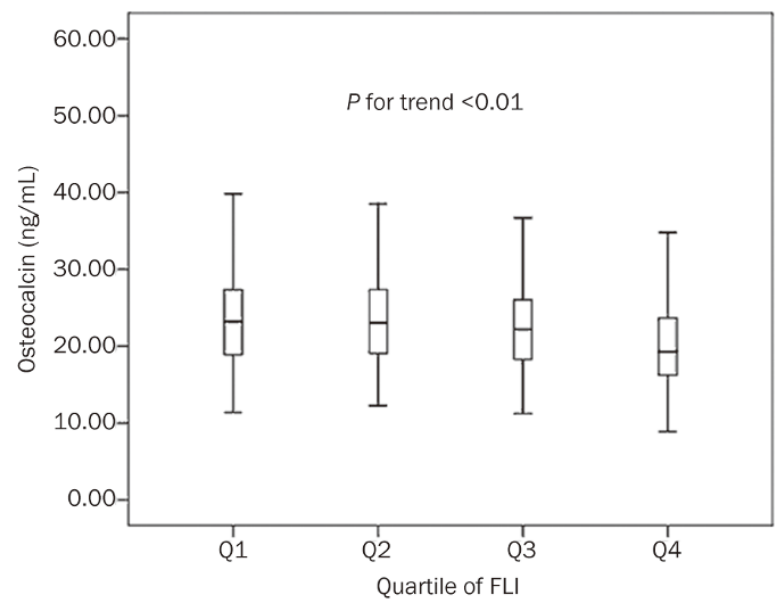

Figure 1. Serum osteocalcin levels decreased with increments in the FLI quartile (Q1, FLI<6.34, $n=183$; Q2, 6.34 $\leq \mathrm{FLI}<12.86, n=183$; Q3, 12.86 $\leq \mathrm{FLI}<26.94, n=184 ; \mathrm{Q} 4, \mathrm{FLI} \geq 26.94, n=183$ ). The bars represent the median value, 25 th percentile, and 75 th percentile for the serum osteocalcin levels, with $P<0.01$ for the trend. identification and intervention can inhibit or reverse the progression of NAFLD to hepatic sclerosis, the timely diagnosis of NAFLD remains hindered by its lack of specific clinical manifestations and the absence of reliable serum biomarkers. Although liver biopsy is the gold standard method used to determine the presence and status of NAFLD, the invasiveness of this method and the risk of complications limit its use in clinical practice. However, B-mode ultrasonography, a noninvasive method used for diagnosing fatty liver diseases, can be widely used in both clinical practice and large cohort surveys.

Osteocalcin is a protein that is secreted by osteoblasts, and it is a marker that reflects the status of bone metabolism. Moreover, osteocalcin has recently been shown to play impor-

Table 2. Independent factors related to FLI as determined by multiple stepwise regression analysis.

\begin{tabular}{lcrr}
\hline Independent variables & Standardized $\beta$ & $t$ & $P$ \\
\hline SBP & 0.106 & 3.750 & $<0.001$ \\
2hPG & 0.119 & 4.219 & $<0.001$ \\
HOMA-IR & 0.351 & 11.521 & $<0.001$ \\
HDL-C & -0.281 & -9.558 & $<0.001$ \\
LDL-C & 0.095 & 3.356 & 0.001 \\
CRP & 0.181 & 6.304 & $<0.001$ \\
Osteocalcin & -0.124 & -4.395 & $<0.001$ \\
\hline
\end{tabular}

Independent variables: age, SBP, DBP, FPG, 2hPG, HbA1c, HLD-C, LDL-C, HOMA-IR, CRP, and osteocalcin.

Abbreviation: 2hPG: 2-h plasma glucose level; CRP: C reactive protein; DBP: diastolic blood pressure; FLI: fatty liver index; FPG: fasting plasma glucose; HbA1c: glycated hemoglobin; HDL-C: high-density lipoprotein cholesterol; HOMA-IR: homeostasis model assessment-insulin resistance; LDL-C: low-density lipoprotein cholesterol; SBP: systolic blood pressure.

Table 3. Binary logistic regression analysis of factors independently associated with NAFLD.

\begin{tabular}{|c|c|c|c|c|}
\hline \multirow[b]{2}{*}{ Models } & \multicolumn{4}{|c|}{ Osteocalcin } \\
\hline & $\beta$ & SEM & OR (95\% Cl) & $P$ \\
\hline Unadjusted & -0.091 & 0.018 & $0.913(0.881-0.946)$ & $<0.001$ \\
\hline $\begin{array}{l}\text { Adjusted Age+BMI+ } \\
\text { waist circumference }\end{array}$ & -0.050 & 0.020 & $0.951(0.915-0.989)$ & 0.012 \\
\hline Adjusted ALT+AST+GGT & -0.078 & 0.019 & $0.925(0.891-0.960)$ & $<0.001$ \\
\hline Full model & -0.051 & 0.022 & $0.951(0.911-0.992)$ & 0.020 \\
\hline
\end{tabular}

Independent variables of full model: age, BMI, waist circumference, osteocalcin, blood pressure (SBP, DBP), blood glucose (FPG, 2hPG, HbA1c), blood lipid (TG, HLD-C, LDL-C), liver enzymes (ALT, AST, GGT), HOMA-IR, CRP.

Abbreviation: 2hPG: 2-h plasma glucose level; ALT: alanine aminotransferase; AST: aspartate aminotransferase; BMI: body mass index; CRP: $\mathrm{C}$ reactive protein; DBP: diastolic blood pressure; FPG: fasting plasma glucose; GGT: gamma-glutamyl transpeptidase; HbA1c: glycated hemoglobin; HDL-C: high-density lipoprotein cholesterol; HOMA-IR: homeostasis model assessment-insulin resistance; LDL-C: low-density lipoprotein cholesterol; SBP: systolic blood pressure; TG: triglyceride. 
tant roles in glucose and fat metabolism. Because glycolipid metabolism disorders are closely associated with the presence of NAFLD, the relationship between osteocalcin and NAFLD has garnered worldwide attention ${ }^{[16-18]}$. Studies of animals fed high fat diets have suggested that the administration of exogenous osteocalcin may help to protect against the development of nonalcoholic steatohepatitis by inhibiting liver steatosis, ballooning degeneration, and fibrosis ${ }^{[3]}$. In human studies, subjects with NAFLD diagnosed through liver biopsy had lower serum osteocalcin concentrations compared to control subjects. Furthermore, serum osteocalcin levels were shown to be independently correlated with degrees of hepatocyte ballooning, which in turn were negatively associated with serum transaminase levels and degrees of insulin resistance ${ }^{[4,19]}$.

Because gender differences have been found in the serum concentrations of osteocalcin, the relationship between serum osteocalcin levels and NAFLD in men has been the focus of several investigations. A study of 1683 Chinese men from Guangzhou revealed that low levels of serum osteocalcin were independently associated with the presence of NAFLD diagnosed through ultrasonography ${ }^{[5]}$. Some studies have shown a close relationship between osteocalcin levels and liver enzymes, and continuous unexplained increases in liver enzymes may be indicative of NAFLD. A cross-sectional study of Spanish men conducted by Fernández-Real et al showed that circulating levels of osteocalcin were negatively associated with ALT and AST levels; in subjects experiencing weight loss, changes in ALT levels were linearly associated with changes in osteocalcin levels $(r=-0.55, P=0.003)^{[20]}$. Additionally, our own previous study of 1558 men also revealed an independent association between serum osteocalcin and ALT levels $^{[21]}$.

Currently, only one cohort study conducted with women from Korea has demonstrated an independent relationship between serum osteocalcin levels and the presence of NAFLD in either premenopausal or postmenopausal women ${ }^{[7]}$. However, in that study, several confounding factors, such as the presence of metabolic disorders and the use of relevant medications, were not taken into account. Thus, in the present study, we chose not to enroll subjects with hyperglycemia or those who were taking either lipid lowering or anti-hypertensive agents. It has been proven that post-menopausal women have a greater risk of developing cardiovascular disease than premenopausal women ${ }^{[22]}$; however, no previous studies have specifically investigated the relationship between serum osteocalcin levels and NAFLD in postmenopausal Chinese women. After adjusting for confounding factors, our study identified an independent association between serum osteocalcin levels and NAFLD, and this association existed both qualitatively and quantitatively. Based on our findings, we speculate that osteocalcin might be involved in regulating lipid metabolism and reducing fat deposition in the liver.

It has been shown that decreased estrogen levels in postmenopausal women lead to an array of metabolic diseases, such as NAFLD. Thus, estrogen may exert a protective effect against NAFLD in humans or animals ${ }^{[23]}$. In addition, serum osteocalcin levels can also be influenced by estrogen. A study of ovariectomized rats showed that estrogen replacement therapy triggered a dose-dependent decrease in serum osteocalcin compared to the serum levels of ovariectomized control rats ${ }^{[24]}$. The present study proved that serum osteocalcin levels were inversely correlated with the presence of NAFLD in postmenopausal women; thus, serum osteocalcin may exert a protective effect against metabolic disorders in postmenopausal women.

The protection provided by osteocalcin against the development of NAFLD might be associated with its ability to lower the degree of insulin resistance ${ }^{[25]}$. Several human studies have shown a negative correlation between serum osteocalcin levels and insulin resistance, as evaluated by HOMAIR; however, these studies have also demonstrated a positive correlation between osteocalcin levels and $\beta$-cell function ${ }^{[26-28]}$. Additionally, a murine study has shown that daily injections of osteocalcin significantly improved glucose tolerance and insulin sensitivity in mice fed either a normal or high fat diet. Furthermore, hepatic steatosis induced by the high fat diet was completely reversed in mice receiving a daily injection of osteocalcin $^{[29]}$. Finally, results from in vitro studies with cultured cells have indicated that osteocalcin can rescue impaired insulin sensitivity through the NF-kB signaling pathway ${ }^{[30]}$.

Our study has some limitations that should be mentioned. First, there are undercarboxylated and carboxylated forms of total serum osteocalcin. Initial animal studies have indicated that undercarboxylated osteocalcin is the biologically active isoform that mediates metabolic functions. However, several recent clinical studies have demonstrated that both undercarboxylated and total osteocalcin were associated with energy metabolism ${ }^{[31,32]}$. Because of the lack of an automated assay to examine the undercarboxylated form, we measured only total serum osteocalcin. Second, all NAFLD patients were diagnosed through ultrasonography, which has low sensitivity for detecting hepatic steatosis. Third, the bone metabolism status of a patient might be a factor influencing the level of serum osteocalcin. In addition, our study population was a subgroup of participants who were previously enrolled in the Shanghai Obesity Study. Our findings must be verified in other populations by studies that use a more accurate method of evaluating a subject's degree of NAFLD and that consider the bone metabolism status of each participant.

\section{Conclusion}

Serum osteocalcin levels were inversely correlated with the presence of NAFLD in postmenopausal Chinese women with normal blood glucose levels.

\section{Acknowledgements}

This work was funded by a project of the National Natural Science Foundation of China (No 81170788), 973 Program of China (№ 2012CB524906), the National Key Technology R\&D Program of China (№ 2012BAI02B03), a grant from Shanghai Health and Family Planning Commission (No 2013ZYJB1001), and a Key Science and Technology of Shanghai Project (No 13XD1403000). We appreciate the efforts of all participants who 
contributed to the sample measurements and data collection.

\section{Author contribution}

Yu-qian BAO and Wei-ping JIA conceived and designed the experiments. Yu-qi LUO, Xiao-jing MA, Ya-ping HAO, Qin XIONG, Yi-ting XU performed the experiments. Xiao-ping PAN provided technical support. Yu-qi LUO and Xiao-jing MA performed the statistical analysis and wrote the paper.

\section{References}

1 Movahed A, Larijani B, Nabipour I, Kalantarhormozi M, Asadipooya $\mathrm{K}$, Vahdat $\mathrm{K}$, et al. Reduced serum osteocalcin concentrations are associated with type 2 diabetes mellitus and the metabolic syndrome components in postmenopausal women: the crosstalk between bone and energy metabolism. J Bone Miner Metab 2012; 30: 683-91.

2 Oosterwerff MM, van Schoor NM, Lips P, Eekhoff EM. Osteocalcin as a predictor of the metabolic syndrome in older persons: a populationbased study. Clin Endocrinol (Oxf) 2013; 78: 242-7.

3 Gupte AA, Sabek OM, Fraga D, Minze LJ, Nishimoto SK, Liu JZ, et al. Osteocalcin protects against nonalcoholic steatohepatitis in a mouse model of metabolic syndrome. Endocrinology 2014; 155: 4697-705.

4 Yilmaz Y, Kurt R, Eren F, Imeryuz N. Serum osteocalcin levels in patients with nonalcoholic fatty liver disease: association with ballooning degeneration. Scand J Clin Lab Invest 2011; 71: 631-6.

5 Liu JJ, Chen YY, Mo ZN, Tian GX, Tan AH, Gao Y, et al. Relationship between serum osteocalcin levels and non-alcoholic fatty liver disease in adult males, South China. Int J Mol Sci 2013; 14: 19782-91.

6 Zhou M, Ma X, Li H, Pan X, Tang J, Gao Y, et al. Serum osteocalcin concentrations in relation to glucose and lipid metabolism in Chinese individuals. Eur J Endocrinol 2009; 161: 723-9.

7 Sinn DH, Gwak GY, Rhee SY, Cho J, Son HJ, Paik YH, et al. Association between serum osteocalcin levels and non-alcoholic fatty liver disease in women. Digestion 2015; 91: 150-7.

8 Bao Y, Ma X, Yang R, Wang F, Hao Y, Dou J, et al. Inverse relationship between serum osteocalcin levels and visceral fat area in Chinese men. Inverse relationship between serum osteocalcin levels and visceral fat area in Chinese men. J Clin Endocrinol Metab 2013; 98: 345-51.

9 World Health Organization. Report of a WHO Consultation: Definition, diagnosis and classification of diabetes mellitus and its complications. Part 1: diagnosis and classification of diabetes mellitus. Geneva: Switzerland, 1999.

10 Hao Y, Ma X, Luo Y, Shen Y, Dou J, Pan X, et al. Serum adipocyte fatty acid binding protein levels are positively associated with subclinical atherosclerosis in Chinese pre- and postmenopausal women with normal glucose tolerance. J Clin Endocrinol Metab 2014; 99: 4321-7.

11 Hao YP, Ma XJ, Luo YQ, Ni J, Dou JX, Hu YQ, et al. Serum vitamin D is associated with non-alcoholic fatty liver disease in Chinese males with normal weight and liver enzymes. Acta Pharmacol Sin 2014; 35: 1150-6.

12 Fan JG. Chinese Liver Disease Association. Guidelines for management of nonalcoholic fatty liver disease: an updated and revised edition. Zhonghua Gan Zang Bing Za Zhi 2010; 18: 163-6.

13 Bedogni G, Bellentani S, Miglioli L, Masutti F, Passalacqua M, Castiglione A, et al. The fatty liver index: a simple and accurate predictor of hepatic steatosis in the general population. BMC Gastroenterol 2006; 6: 33.

14 Targher G, Day CP, Bonora E. Risk of cardiovascular disease in patients with nonalcoholic fatty liver disease. N Engl J Med 2010; 363: 1341-50.

15 Gao X, Fan JG. Diagnosis and management of non-alcoholic fatty 1iver disease and related metabolic disorders: consensus statement from the Study Group of Liver and Metabolism, Chinese Society of Endocrinology. J Diabetes 2013; 5: 406-15.

16 Yang R, Ma X, Pan X, Wang F, Luo Y, Gu C, et al. Serum osteocalcin levels in relation to metabolic syndrome in Chinese postmenopausal women. Menopause 2013; 20: 548-53.

17 Díaz-López A, Bulló M, Juanola-Falgarona M, Martínez-González MA, Estruch R, Covas MI, et al. Reduced serum concentrations of carboxylated and undercarboxylated osteocalcin are associated with risk of developing type 2 diabetes mellitus in a high cardiovascular risk population: a nested case-control study. J Clin Endocrinol Metab 2013; 98: 4524-31.

18 Sarkar PD, Choudhury AB. Relationships between serum osteocalcin levels versus blood glucose, insulin resistance and markers of systemic inflammation in central Indian type 2 diabetic patients. Eur Rev Med Pharmacol Sci 2013; 17: 1631-5.

19 Aller R, Castrillon JL, de Luis DA, Conde R, Izaola O, Sagrado MG, et al. Relation of osteocalcin with insulin resistance and histopathological changes of non alcoholic fatty liver disease. Ann Hepatol 2011; 10 : 50-5.

20 Fernández-Real JM, Ortega F, Gómez-Ambrosi J, Salvador J, Frühbeck G, Ricart W. Circulating osteocalcin concentrations are associated with parameters of liver fat infiltration and increase in parallel to decreased liver enzymes after weight loss. Osteoporos Int 2010; 21 : 2101-7.

21 Dou J, Ma X, Fang Q, Hao Y, Yang R, Wang F, et al. Relationship between serum osteocalcin levels and non-alcoholic fatty liver disease in Chinese men. Clin Exp Pharmacol Physiol 2013; 40: 282-8.

22 Auro K, Joensuu A, Fischer K, Kettunen J, Salo P, Mattsson H, et al. A metabolic view on menopause and ageing. Nat Commun 2014; 5: 4708.

23 Lavoie JM, Pighon A. NAFLD, estrogens, and physical exercise: The Animal Model. J Nutr Metab 2012; 2012: 914938.

24 Williams DC, Paul DC, Black $\sqcup$. Effects of estrogen and tamoxifen on serum osteocalcin levels in ovariectomized rats. Bone Miner 1991; 14: 205-20.

25 Utzschneider KM, Kahn SE. The role of insulin resistance in nonalcoholic fatty liver disease. J Clin Endocrinol Metab 2006; 91: 4753-61.

26 Chen L, Li Q, Yang Z, Ye Z, Huang Y, He M, et al. Osteocalcin, glucose metabolism, lipid profile and chronic low-grade inflammation in middle-aged and elderly Chinese. Diabet Med 2013; 30: 309-17.

27 Bulló M, Moreno-Navarrete JM, Fernández-Real JM, Salas-Salvadó J. Total and undercarboxylated osteocalcin predict changes in insulin sensitivity and $\beta$ cell function in elderly men at high cardiovascular risk. Am J Clin Nutr 2012; 95: 249-55.

28 Lucey AJ, Paschos GK, Thorsdottir I, Martínéz JA, Cashman KD, Kiely M. Young overweight and obese women with lower circulating osteocalcin concentrations exhibit higher insulin resistance and concentrations of C-reactive protein. Nutr Res 2013; 33: 67-75.

29 Ferron M, McKee MD, Levine RL, Ducy P, Karsenty G. Intermittent injections of osteocalcin improve glucose metabolism and prevent type 2 diabetes in mice. Bone 2012; 50: 568-75.

30 Zhou B, Li H, Xu L, Zang W, Wu S, Sun H. Osteocalcin reverses endoplasmic reticulum stress and improves impaired insulin sensitivity secondary to diet-induced obesity through nuclear factorkappaB signaling pathway. Endocrinology 2013; 154: 1055-68.

31 Pittas AG, Harris SS, Eliades M, Stark P, Dawson-Hughes B. Association between serum osteocalcin and markers of metabolic phenotype. J Clin Endocrinol Metab 2009; 94: 827-32.

32 Hu WW, Ke YH, He JW, Fu WZ, Liu YJ, Chen D, et al. Serum osteocalcin levels are inversely associated with plasma glucose and body mass index in healthy Chinese women. Acta Pharmacol Sin 2014; 35: 1521-6. 